\title{
Plan de gestión del consumo residencial para la Empresa Eléctrica Regional Centro Sur C.A.
}

Ricardo Medina ${ }^{1}$

ricardodavid_m@hotmail.com

\section{Resumen}

La gestión de la demanda eléctrica se presenta como una alternativa a la crisis energética mundial, considerando el actual problema energético que atraviesa nuestro país, es indispensable dotar al consumidor final de planes concretos de ahorro de energía, cuyos beneficios directos se reflejen en un menor consumo de energía y por lo tanto una menor facturación.

La crítica situación medioambiental que enfrenta nuestra sociedad, requiere de un uso más eficiente de los recursos disponibles, es imperativo dotar a nuestra región de herramientas para realizar un ahorro en cada casa; sabiendo que la suma de estos 'pequeños' ahorros permitirá mantener fuera del despacho económico las centrales de tecnología obsoleta que contaminan el ambiente con costos de operación elevados.

Palabras clave: Gestión de la Demanda Eléctrica, Demand Side Maganament, Eficiencia Energética, Bonos de Carbono.

\section{Abstract}

Demand Side Management represents an alternative to the global energy crisis, knowing the current energy deficit problem of our country, it is urgent to propose concrete plans for energy savings for the final consumer, which direct benefits are reflected in a lower consumption and final monthly account.

Our society is facing a critical environmental plight, it requires more efficient use of available resources, it is imperative to provide our region with tools for every housebold savings, the sum of these "small savings" will be kept out, of economic dispatch, the obsolete plants whit high operating costs and extremely polluting.

Keywords: Demand Side Management, Energy Efficiency, Certified Emission Reductions, Coal Credits.

1 R. Medina. Estudiante del programa de doctorado de la Universidad Nacional de San Juan - Argentina. 


\section{Introducción}

El presente documento es un resumen de la investigación realizada como tesis de grado para la obtención del título de Ingeniero Eléctrico dentro del convenio existente entre la Universidad Politécnica Salesiana sede Cuenca y la Empresa Eléctrica Regional Centro Sur. Esta investigación se ejecutó en la ciudad de Cuenca durante el periodo comprendido entre agosto de 2008 y julio de 2009, empleando encuestas y mediciones para definir los usos finales de la energía.

\subsection{Alcance y objetivos}

\subsubsection{Alcance}

El presente proyecto busca determinar acciones concretas que permitan el uso eficiente de energía por parte de clientes residenciales del cantón Cuenca servidos por la CENTROSUR.

\subsubsection{Objetivo general}

Determinar acciones concretas y prácticas para que el cliente final de la CENTROSUR pueda obtener un consumo eficiente de energía eléctrica.

\subsubsection{Objetivos específicos}

- Realizar la caracterización del consumo del sector residencial; determinar las recomendaciones para lograr un consumo más eficiente de la energía y definir la propuesta de un plan de incentivos para que los clientes residenciales de la CENTROSUR reduzcan su consumo.

- Realizar el análisis económico del impacto de las medidas del "Plan de gestión del consumo residencial” para la CENTROSUR y para los clientes residenciales.

\section{Determinación de la muestra estadística para la investigación}

Para realizar un plan de uso eficiente de energía eléctrica es necesario conocer el uso final de este recurso por parte de los clientes residenciales.

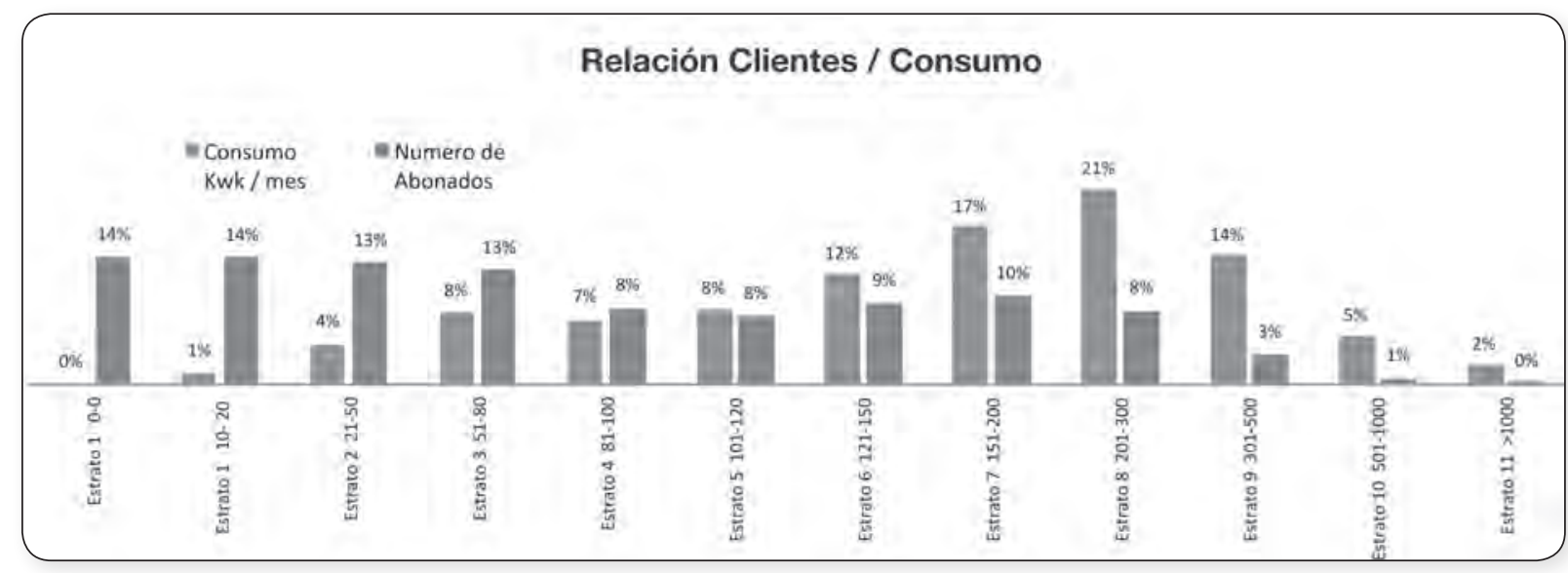

Gráfico 1. Relación entre consumo y clientes residenciales del total del Sistema CENTROSUR. Fuente: Elaboración propia a base de la información de la CENTROSUR. 
En el plan de gestión del consumo residencial se observa que el 70\% de los clientes están en los estratos $0-5$, representando tan solo el $29 \%$ del consumo, en comparación de los estratos 6-11 que corresponden al 71\% del consumo.

\subsection{Muestreo de poblaciones}

\subsubsection{Muestreo por conglomeración}

Éste se lo realiza en áreas territoriales muy grandes. Consiste en dividir el territorio en varias regiones menores para seleccionar las más representativas, y que tengan características comunes a la mayoría de la población; en el caso de la CENTROSUR, su área de cobertura es muy extensa y se requiere aplicar un muestreo de este tipo, las divisiones territoriales existentes a nivel político pueden ser usadas con gran eficiencia, debido a su existencia anterior y facilidad de ubicación.

Con esta precisión es necesario realizar una determinación geográfica e identificación de las subdivisiones sobre las cuales se realizará el estudio.

\subsubsection{Delimitación geográfica}

Es necesario determinar las conductas, comportamientos y hábitos de consumo de los clientes residenciales de la CENTROSUR; por lo tanto, se requiere definir un porcentaje de la población para realizar un análisis de su consumo, y encontrar los hábitos de consumo del conjunto de clientes residenciales del cantón Cuenca servido por la CENTROSUR.

Considerando la población del sistema servido por la CENTROSUR constatamos que aproximadamente el $89 \%$ de los clientes son residenciales y su consumo equivale a cerca del $49 \%$ de la energía facturada por la empresa.
Clientes urbanos del cantón Cuenca respecto al Total del Sistema CENTROSUR

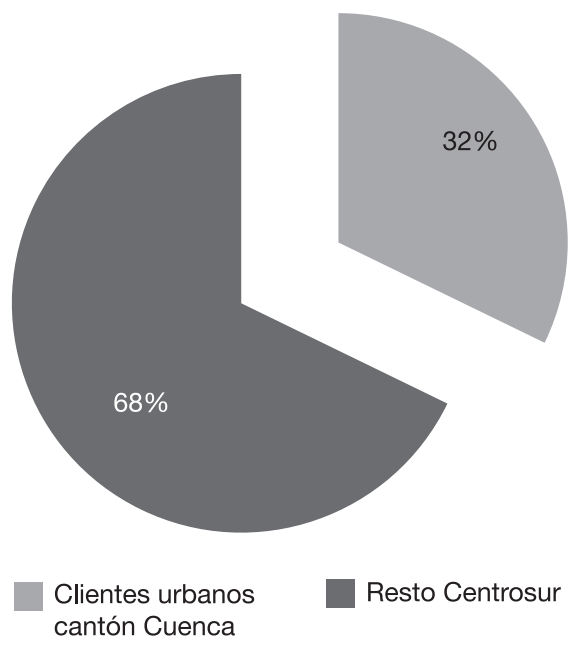

Gráfico 2. Clientes urbanos del cantón Cuenca respecto al total del sistema CENTROSUR.

\subsubsection{Número de muestras}

Para determinar el tamaño de una muestra se requiere conocer ciertos parámetros tales como: nivel de confianza deseado, margen de error tolerado y variabilidad de la población.

El nivel de confianza es la certeza de que la muestra representa verdaderamente al universo que se estudia; se elige entre 0 y $100 \%$ pero los valores más comunes se dan entre un 95 y 99\%, con éstos se obtiene valores ' $z$ ' correspondientes. Al 95\% le corresponde un valor de ' $z$ ' de 1,96 , y a 99\% 2,58; el error permitido es la cantidad que se suma o resta a la media muestral para determinar los extremos de los intervalos de confianza. En definitiva, es el error que se está dispuesto a tolerar; si el error es grande se tendrá una muestra pequeña y si se desea un error pequeño la muestra será más grande. En la presente investigación corresponde a la máxima diferencia en el consumo entre estratos. 
La variabilidad de la población se determina mediante la desviación estándar, este valor se puede obtener de estudios anteriores o información previa. En este caso se empleará la desviación estándar de los consumos por estratos.

$$
n=\left(\frac{Z \cdot S}{E}\right)^{2}
$$

3. $n=\left(\frac{1.96 \cdot 540901,9}{100000}\right)^{2}=112,39 \approx$

\section{3 mediciones}

Ecuación 1. Tamaño de la muestra porcentual

Donde:

n: Tamaño de la muestra.

z: Valor normal estándar que corresponde al nivel de confianza deseado.

s: Desviación estándar estimada de la población.

E: Máximo error permitido.

\subsubsection{Número de muestras por grupo de consumo}

Una vez determinado el tamaño de la muestra debe conocerse cuantas muestras realizar en cada tipo de tarifa (industrial, residencial, comercial, alumbrado público, otros). Esta distribución de las muestras se realiza proporcionalmente a la participación de cada tarifa en el total de clientes de la distribuidora, el cálculo de las muestras por tarifa explica esta distribución, las fracciones de muestra se deben redondear al inmediato superior.

$$
n_{\text {m/estrato }}=n_{\text {mtotal }} \cdot \frac{n_{\text {cons_estrato }}}{N}
$$

Ecuación 2. Cálculo de las muestras por tarifa

Donde:

$n_{m / \text { estrato }}:$ Número de muestras por tarifa.

$n_{\text {motal }}: \quad$ Número de muestras totales.

$n_{\text {cons estrato }}$ : Consumo total de los clientes de la tarifa.

$N$ : $\quad$ Consumo total de los clientes servidos por la empresa distribuidora.

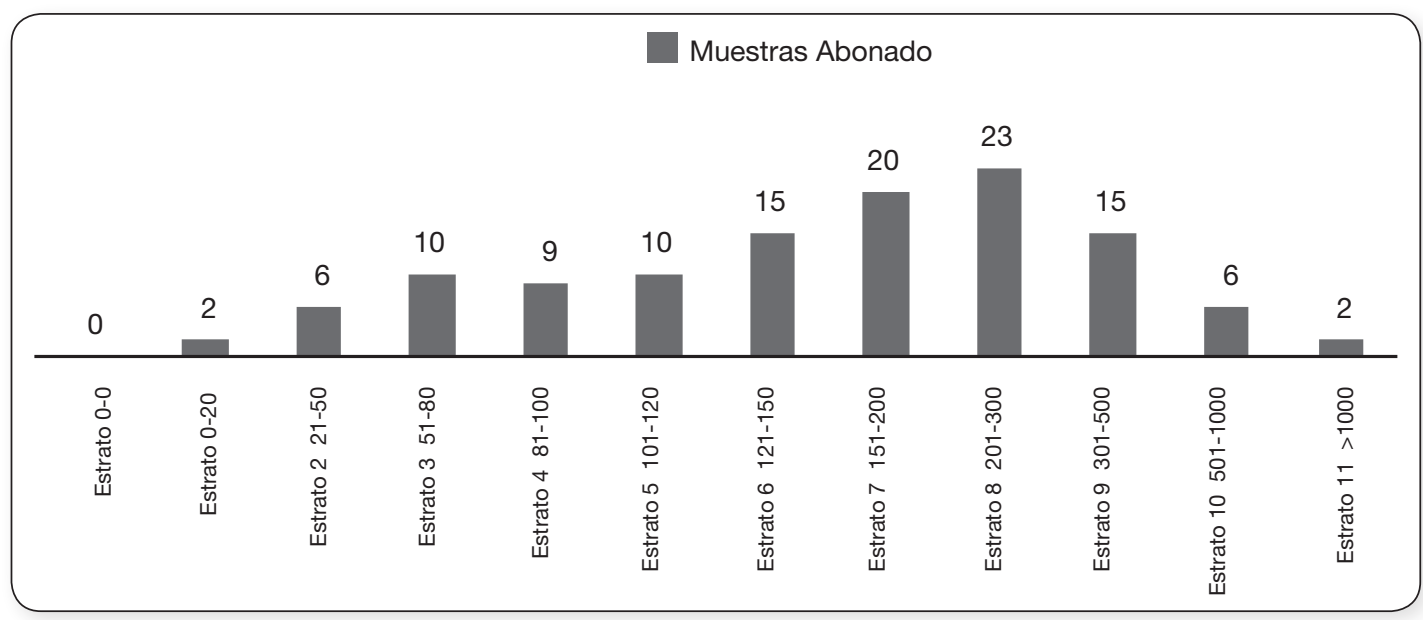

Gráfico 3. Número de mediciones por Estratos Fuente: Elaboración propia. 
Esta distribución de la cantidad de las muestras permite realizar más mediciones y encuestas en las tarifas con mayor significancia de consumo, permitiendo una mayor confianza de los resultados.

\section{Plan de gestión de consumo residencial}

\subsection{Eficiencia energética en el mundo actual}

En la situación mundial actual, el uso eficiente de los recursos es un compromiso y responsabilidad de todos; la eficiencia energética (EE) está definida como "conjunto de acciones que permiten optimizar la relación entre la cantidad de energía consumida y los productos y servicios finales obtenidos. Esto se puede lograr a través de la implementación de diversas medidas e inversiones a nivel tecnológico, de gestión y de hábitos culturales en la comunidad”.

La energía se consume de una manera ineficiente, existen pocas horas en el día en las cuales se realiza un consumo elevado, el sistema eléctrico debe estar sobre dimensionado en las etapas de generación, transporte y distribución, lo cual representa una inversión adicional. Durante la mayor parte del día, la capacidad del sistema es subutilizado, lo cual, además del impacto económico, afecta la eficiencia. A continuación se presenta la curva Monótona de Demanda, en la cual se evidencia que durante muy pocas horas al año se consume una gran cantidad de energía.

\subsection{Plan de gestión de la demanda}

Desde la crisis petrolera de la década del setenta, se llevan a cabo investigaciones sobre diversificación de las fuentes energéticas y el

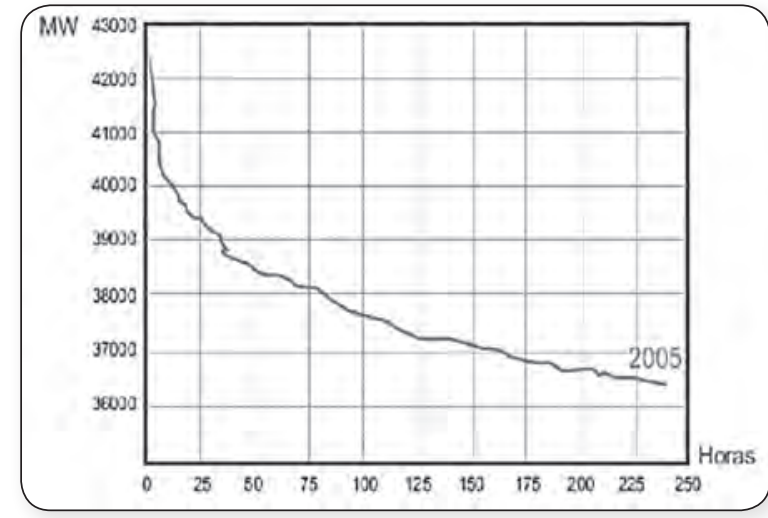

Gráfico 4. Monótona de Demanda del sistema peninsular español, se presenta las 240 horas de mayor consumo del año 2005.

Fuente: Red Eléctrica Española REE.

uso eficiente de la energía. En varios países se desarrollan planes de Gestión de la Demanda Eléctrica GDE (o Demand Side Management -DSM-); la Gestión de la Demanda Eléctrica (GDE) consiste en la implementación de políticas y medidas que motiven a los usuarios finales de energía eléctrica a reducir su consumo, cambiando hábitos de consumo o reemplazando equipos eléctricos por artefactos de mayor eficiencia.

Es necesario realizar una puntualización. Los términos ahorro energético y eficiencia energética pueden parecer iguales pero en esencia indican dos realidades distintas; el ahorro de energía significa 'apagar las luces' sacrificando actividades o procesos, este es el caso de los racionamientos de energía; la eficiencia energética se enfoca a reducir el consumo de energía sin afectar la calidad del servicio o la periodicidad de ciertos procesos mediante el uso de equipos de menor consumo que cumplan las mismas funciones del equipo al que sustituyen, como se da en el caso de los focos incandescentes frente a las lámparas fluorescentes compactas que iluminan igual pero consumen una fracción de la energía que las incandescentes. 


\subsection{Beneficios para la empresa comercializadora}

Para la CENTROSUR, la implementación de un PGD significa una reducción en los ingresos que obtenga por concepto de la venta de energía; sin embargo, los beneficios serán: económicos y de imagen corporativa, ya que se la presentará a la CENTROSUR comprometida con la conservación del planeta al plantear a sus clientes una estrategia para combatir el cambio climático al reducir el consumo de energía.

Un plan de gestión de demanda implica gastos o reducción en las ventas de energía, pero la empresa comercializadora puede obtener los siguientes beneficios:

a. Reducción de la emisión de gases de efecto invernadero (ambiental).

b. Imagen pública (marketing).

c. Calidad de servicio (regulaciones).

d. Incentivos por parte del agente regulador (regulaciones).

e. Servir a nuevos clientes sin necesidad de modificar la infraestructura existente (técnico).

\subsection{La demanda en los planes de gestión del consumo}

Tradicionalmente, se ha considerado a la demanda de energía eléctrica como una variable determinada por "factores externos, fuera de control y que hace falta predecir (MEER, 2008: 16)", frente a lo cual es necesario un cambio en los esquemas; las perspectivas sobre la demanda en una GDE apuntan más bien a controlar y organizar el consumo de manera que sea posible la mayor eficiencia en la producción, transporte y distribución.
La GDE plantea que la demanda puede ser reducida y controlada

Controlada o ajustada: desplazando ciertos consumos de las horas pico a otras horas (demanda elástica).

Reducida: si se sustituyen artefactos eléctricos por otros de mayor eficiencia, o cambiando hábitos de consumo como apagar artefactos no usados, o usarlos en forma eficiente.

Un plan de GDE tiene como meta que los clientes de una comercializadora realicen su consumo de energía de manera eficiente, elevando el factor de carga; esto se puede obtener disminuyendo el consumo en las horas pico o en su defecto desplazando ciertos consumos a otros momentos del día, especialmente en los llamados 'valles de carga', en la Figura 5: Puntos característicos de la Curva de Carga, se observa que el máximo consumo del día se produce a las $20 \mathrm{~h} 00$, si se desplazara este consumo a horas de la noche después de las $22 \mathrm{~h} 00$ hasta las $06 \mathrm{~h} 00$ o a partir de las $09 \mathrm{~h} 00$ hasta las $16 \mathrm{~h} 00$, el factor de carga mejora.

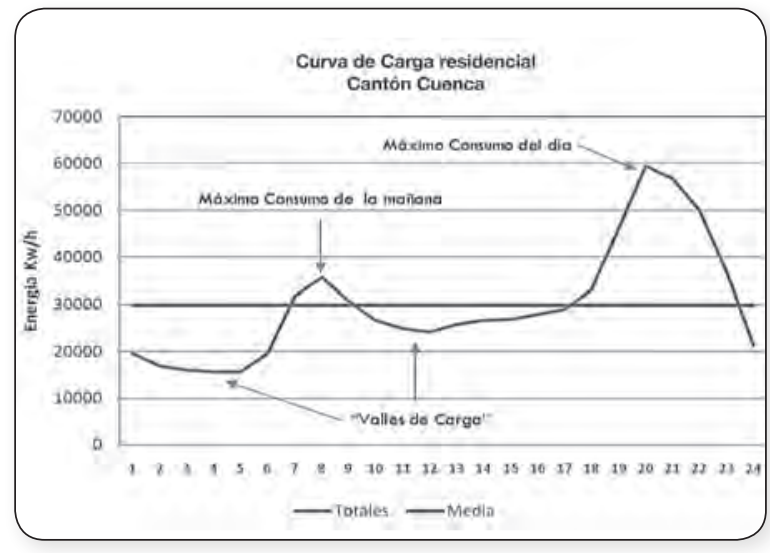

Gráfico 5. Puntos característicos de la Curva de Carga. Fuente: Elaboración propia, basándose en la Curva de Carga Residencial del cantón Cuenca. 


\subsection{Estrategias en un Plan de gestión de consumo de energía}

Las estrategias empleadas en una GDE son fundamentalmente: informativas, investigación, administrativas y financieras ${ }^{2}$.

a. Informativas: están enfocadas en difundir información sobre el uso eficiente de la energía, crear conciencia y compromiso entre los usuarios finales.

b. Investigación: desarrollar tecnologías eficientes, adaptar descubrimientos y avances a las realidades particulares en donde se aplica una GDE.

c. Administrativas: estas estrategias se basan en establecer normativas, regulaciones, certificaciones, están dirigidas a motivar y regular la el plan de eficiencia energética.

d. Financieras: es necesario incentivar las inversiones en planes y tecnologías, premiando la reducción de consumo o brindando el financiamiento para que los clientes puedan adquirir equipos eficientes.

La presente investigación se enfocó básicamente en estrategias informativas y abarcará tópicos financieros dirigidos a incentivos económicos para los clientes.

\subsection{Acciones del Plan de gestión de consumo de energía}

Para llevar a cabo un plan de GDE es necesario definir las acciones que se aplicarán; en general, estas estrategias son a 'ganar-ganar'; es decir, gana el cliente al reducir su consumo, gana la comercializadora al mejorar, entre otros parámetros, su factor de carga y al re- ducir el consumo en horas pico, gana el país porque reduce la producción de energía y se puede servir a otros sectores con la misma infraestructura ya existente.

Se puede clasificar este tipo de acciones según su inversión en dos grupos; las acciones sin costo de inversión para el cliente y las acciones que involucren inversión.

\subsubsection{Acciones sin inversión}

Estas acciones implican un cambio en las costumbres de los clientes y son enfocadas en las campañas de información. Se requiere un cambio en la forma de usar la energía, la primera cuestión que se debe analizar es la adecuada relación entre la energía consumida y el trabajo realizado.

En general, los clientes son motivados a ahorrar o usar energía de forma eficiente para reducir el pago en su planilla. Apagando artefactos eléctricos en desuso y utilizando eficientemente los electrodomésticos se pueden obtener mayores reducciones.

\subsubsection{Acciones con inversión}

Básicamente se enfocan en realizar cambios en las construcciones para mejorar aislamientos térmicos o sustituir equipos eléctricos por otros más eficientes, lo cual requiere una inversión. Es necesario analizar la inversión inicial para ejecutar estas acciones y el beneficio reportado, se las clasificará según el tiempo en el cual se recuperará la inversión, con este criterio se puede tener: acciones de retorno al corto plazo (short payback time actions), acciones de retorno al medio plazo (medium

2 Las estrategias mencionadas fueron recogidas de los siguientes documentos: "Gestión de la demanda eléctrica, un método eco-energético"; M. Torrent y M.A. Larrayoz; Dpto. Ingeniería Eléctrica. Universidad Politécnica de Cataluña, "Demand Side Management for residential and commercial end-users"; by PhD bonneville, Eric; rialhe, Anne; Aere-France. 
payback time mesures) y acciones de retorno al largo plazo (long payback time mesures).

Estas acciones a corto, mediano y largo plazo dependen de los hábitos, confort y estilo de vida de los clientes determinados de una u otra forma por la cultura del medio.

\subsubsection{Acciones de retorno a corto plazo}

Son típicamente acciones técnicas simples que permiten ahorro inmediato, con inversiones pequeñas que pueden ser fácilmente recuperadas directamente y sus resultados se ven reflejados con la reducción de la planilla de energía.

Por lo general son:

- Aprovechamiento de la luz natural, mejorando la distribución de zonas de trabajo.

- Mejoramiento de aislamientos térmicos en construcciones y viviendas.

- Eliminación del consumo en stand-by de los equipos.

- Colocación de sistemas de apagado y encendido automático de calefacción, ventilación e iluminación en pasillos, baños y áreas de circulación intermitente, esto puede reducir el consumo de energía de estas zonas entre un 25-50\% (Alpheo, 2005).

\subsubsection{Acciones de retorno a mediano plazo}

Permiten un mayor ahorro de energía pero requieren una mayor inversión, entre las medidas a medio plazo están:

- Sustitución de iluminación incandescente por lámparas fluorescentes compactas LFC u otras tecnologías de iluminación. Este proyecto se lleva a cabo en Ecuador, en la actualidad las acciones son impulsadas por el MEER.

- Cambio de energía usada en el calentamiento de agua. Se puede optar por adquirir un calentador solar de agua en paralelo con un calentador de agua de Gas Licuado de Petróleo (GLP) de respaldo.

- Desplazar el uso de equipos no prioritarios en las horas pico. Este consumo se realiza en horas de menor demanda (valles de carga), por ejemplo, el lavado de ropa o vajilla; esta acción es muy útil si los precios de la energía son menores en las horas 'valle', de esta manera el cliente colabora con la reducción del pico de carga. Para implementar estas medidas es necesario que exista un sistema de medición con registro horario, esta acción depende de la decisión gubernamental ya que exige la adquisición de equipos con un costo elevado.

\subsubsection{Acciones de retorno a largo plazo}

Se enfocan sobre todo a cambios en las estructuras de las viviendas con un alto costo de inversión inicial y, consecuentemente, un largo periodo de recuperación de la inversión, entre ellas tenemos:

- Mejorar aislamientos internos y externos en una vivienda. Esto evita la perdida de calor en invierno y mantiene la temperatura interior agradable en verano. Estas acciones modifican estructuralmente la vivienda, se pueden realizar en la etapa de construcción o cuando la vivienda ya está habitada, en ambos casos las viviendas tienen un costo mayor que una similar sin aislamientos pero el beneficio se presenta al reducir el consumo de energía en refrigeración o calefacción.

- Reemplazo de electrodomésticos por unidades más eficientes. Se enfoca en la sustitu- 
ción de equipos que estén cerca de cumplir su vida útil o en su defecto cuando se desea comprar electrodomésticos nuevos. El principal limitante en estos casos es el precio de compra ya que un aparato más eficiente tiene un costo mayor.

\section{Acciones del Plan de gestión de demanda para el cantón Cuenca}

Se presentan algunas soluciones y sus correspondientes métodos de aplicación para reducir el consumo en el sector residencial del cantón Cuenca. Las opciones elegidas en esta investigación tienen sustento económico:

- Campaña informativa.

- Sustitución de calentadores eléctricos por calentadores solares.

- Participación de la CENTROSUR en el mercado de bonos de carbono.

- Retraso de inversiones en expansión del Sistema de Subtransmisión y Distribución.

Cada acción mencionada tiene su análisis que incluye: objetivos, actores, barreras para la implementación, beneficios, financiamiento, etapas de implementación, indicadores y sustento económico.

Conjuntamente con cada propuesta se incluye una evaluación económica detallada de los beneficios al aplicar estas acciones.

\subsection{Campaña informativa}

Pretende difundir a los clientes residenciales concejos de ahorro de energía en el hogar, uso eficiente de energía y concientización sobre la importancia de realizar un consumo consiente y eficiente.
Esta línea de acción no involucra ningún gasto para el cliente final ya que se plantea el cambio de costumbres en cuanto al consumo de energía; la difusión se realizaría por parte de la CENTROSUR.

\subsubsection{Objetivos}

a. Establecer mecanismos para que los clientes residenciales del cantón Cuenca realicen su consumo de energía considerando la eficiencia energética.

b. Realizar campañas informativas sobre eficiencia energética en el área educativa.

c. Dar indicadores económicos al mercado y a los clientes para que realicen su consumo energético más eficientemente.

\subsubsection{Actores}

a. Primarios: los clientes residenciales del cantón Cuenca.

b. Secundarios: Empresa Eléctrica Regional CENTROSUR, Ministerio de Electricidad y Energías Renovables.

\subsubsection{Barreras para implementación de la campaña informativa sobre eficiencia energética}

En realidad no existen dificultades externas para la difusión de una campaña de eficiencia energética, el único limitante serían los costos que tendría; a nivel de clientes los costos de adquisición de equipos eficientes y mejoras en el hogar, la motivación para cumplir las recomendaciones será la reducción de consumo y el ahorro económico mensual.

\subsubsection{Beneficios}

Realizar la campaña informativa sobre eficiencia energética. Esta acción repercutirá en 
las costumbres y hábitos de consumo, por lo que tendrá los siguientes beneficios:

\section{a. Económicos:}

- Reducción del valor de las planillas.

- Reducción en el consumo de derivados de petróleo para la generación termoeléctrica.

\section{b. Técnicos:}

- Reducción del consumo diario, esta reducción ayudará a mejorar el factor de carga del sistema y reducirá la producción de energía a lo largo del día.

- Mejora la el uso de las redes actuales de distribución y aumenta la eficiencia del sistema de distribución de la CENTROSUR.

\section{c. Ambientales:}

Reducción de los gases de efecto invernadero ya que se requiere menor producción de energía eléctrica.

\section{d. Marketing:}

Si la CENTROSUR brinda información sobre cómo reducir el consumo, el cliente percibe una imagen de interés en sí. Esto es llamado "satisfacción del cliente", por parte de la empresa; además del compromiso con la situación medioambiental.

\subsubsection{Formas de financiamiento}

Esta campaña puede recibir financiamiento de la empresa privada o directamente de la CENTROSUR; la inversión se enfocará a la edición y publicación por medios impresos, televisión y radio sobre estrategias para reducir el consumo energético y que beneficios se obtendrán.

\subsubsection{Etapas de implementación}

a. Compromiso con socios estratégicos que para la edición y transmisión de la campaña informativa.

b. Difusión publicitaria de la campaña en medios de comunicación e iniciar campañas a nivel educativo en escuelas, colegios, universidades y grupos de consumidores, se puede actualizar la campaña a instituciones educativas del país que fue usada con el nombre de $\mathrm{PAE}^{3}$.

c. Establecimiento de acuerdos voluntarios con clientes o grupos de clientes para reducción de consumo, mediante búsqueda de créditos y capacitación sobre uso eficiente de energía y nuevas tecnologías.

d. Implementación de medidores 'inteligentes' o de contadores de registro que faciliten una tarifación horaria.

En el ámbito educativo podemos mencionar campañas educativas realizadas en otros países y que pueden ser estudiadas y aplacadas en nuestro medio:

\section{a. KIOTO EDUCA ${ }^{4}$}

Kioto Educa es un programa de educación ambiental dirigido a los centros educativos con la intención de sensibilizar al alumnado y al profesorado sobre la necesidad de ahorrar energía y lucha contra el cambio climático, llevado a cabo por la junta de Andalucía en España.

3 En mayo de 2001 se inicio el programa de ahorro de energía (PAE), el cual capacitó a docentes en el país de manera que se difundiera en las escuelas del país, esta campaña a nivel de educación básica se llamó "Guardián de Luz".

4 Tomado y adaptado de la página electrónica: http://www.kiotoeduca.org/ 


\section{b. CHILECTRA}

La empresa chilena Chilectra ${ }^{5}$, dentro de sus propuestas para lograr la eficiencia energética (EE), ha emprendido varios proyectos educativos tales como:

- Diplomado en eficiencia energética, en convenio con la Universidad Mayor de Chile, esta propuesta busca capacitar a profesionales en uso eficiente de la energía eléctrica.

- Concurso de Tesis o Memorias de Pregrado y Posgrado en Eficiencia Energética, esta propuesta se lleva a cabo desde $2007^{6}$ y busca incentivar la investigación en eficiencia energética.

\subsubsection{Indicadores}

- Relación de consumo por habitante.

- Relación de consumo por cliente.

- Cantidad de compromisos voluntarios firmados con grupos de consumidores.

- Cantidad de personar capacitadas mediante los programas educativos.

\subsection{Sustitución de calentadores eléctricos de agua por calentadores solares}

El calentamiento de agua constituye un importante rubro dentro del consumo de energía de los clientes residenciales del cantón Cuen$\mathrm{ca}^{7}$, por lo cual se realiza un análisis de las posibilidades de usar calentadores solares.

La actual situación económica del país y los subsidios a la energía eléctrica y al GLP son una barrera para la utilización de calentadores solares, pero esta alternativa es una muy buena opción por su tasa de retorno de inversión y beneficios ambientales.

En caso de la eliminación de los subsidios mencionados se presenta como una gran opción para los clientes residenciales la colocación de calentadores solares, pues reduce el consumo de energía eléctrica o gas, los sistemas térmico-solares necesitan un respaldo para calentar el agua cuando la climatología no lo permita usar.

\subsubsection{Objetivos}

Sustitución de equipos de calentamiento de agua eléctricos por sistemas termo-solares.

\subsubsection{Actores}

a. Primarios: los clientes residenciales del cantón Cuenca; cualquier local que requiera agua caliente para aplicaciones sanitarias.

b. Secundarios: Empresa Eléctrica Regional Centro Sur, fundaciones, ONG, comercios que puedan proveer los sistemas de calentamiento solar.

\subsubsection{Barreras para implementación del sistema de termo-solar}

En nuestra sociedad existen algunas trabas para implementar proyectos que involucren energías renovables como el caso de los sistemas termo-solares, entre estas dificultades tenemos:

- Ausencia de políticas que incentiven la utilización de energías alternativas o renovables.

5 La página electrónica es: http://www.chilectractiva.cl

6 La información sobre este concurso se puede revisar en: http://www.chilectractiva.cl/link.exe/Noticias/Premios/1561\#

7 En esta investigación se determinó que el calentamiento de agua representa alrededor del $7 \%$ del consumo mensual y un $26 \%$ del consumo en el consumo de la hora pico de la mañana. 
- Falta de recursos económicos para realizar las inversiones.

- Falta de empresas y profesionales en la localidad que ofrezcan estos servicios.

- Resistencia al uso de nuevas tecnologías de construcción y diseño de los domicilios que incluyan energías renovables.

- Subsidios a la energía eléctrica y al GLP.

Según la información colectada en las encuestas, se conoce que un 34\% de los calentadores de agua tienen más de 4 años de uso; esto representa una oportunidad de cambio importante, ya que se puede ofrecer a los clientes la posibilidad de financiar un equipo termo-solar. En la encuesta se preguntó: ¿̇i usa energía eléctrica para calentar el agua cambiaría calentadores solares, si la CENTROSUR le diera facilidades o financiamiento? Un $83 \%$ de los encuestados respondieron positivamente.

Con una adecuada campaña y buscando socios estratégicos que puedan proveer estos equipos se sustituirían calentadores eléctricos por sistemas termo-solares.

\subsubsection{Beneficios}

Aplicar esta técnica de calentamiento de agua proporciona beneficios económicos, técnicos, ambientales y de imagen para la CENTROSUR.

\section{a. Económicos}

- Reducción del valor de las planillas ya que el calentamiento de agua representa el 6,5\% del consumo de energía en el cantón Cuenca.

- Creación de fuentes de trabajo y movilización de la economía ya que se requiere técnicos para la instalación, puntos de venta de materiales.

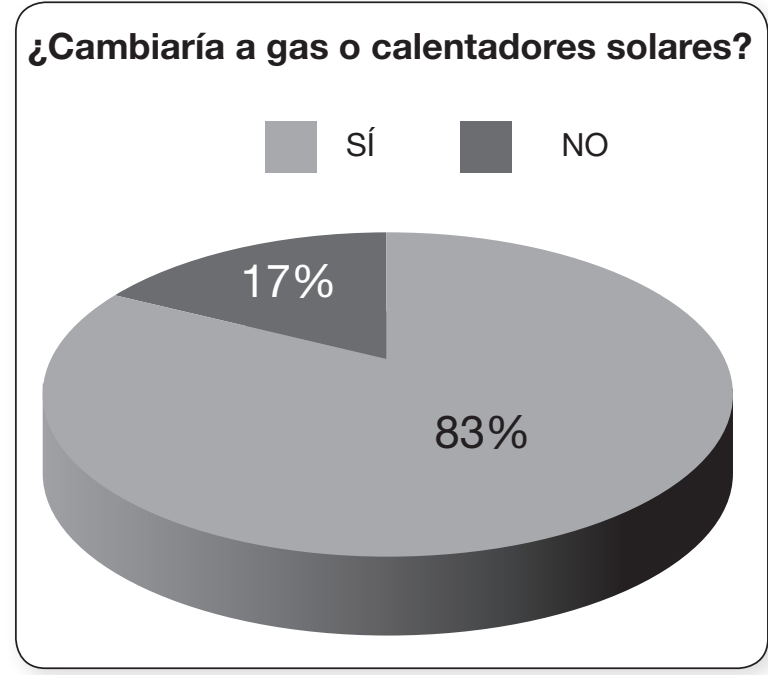

Gráfico 6. Opinión del cliente sobre sustituir su sistema de calentamiento de agua por sistemas solares.

Fuente: Elaboración propia a partir de la información de la encuesta.

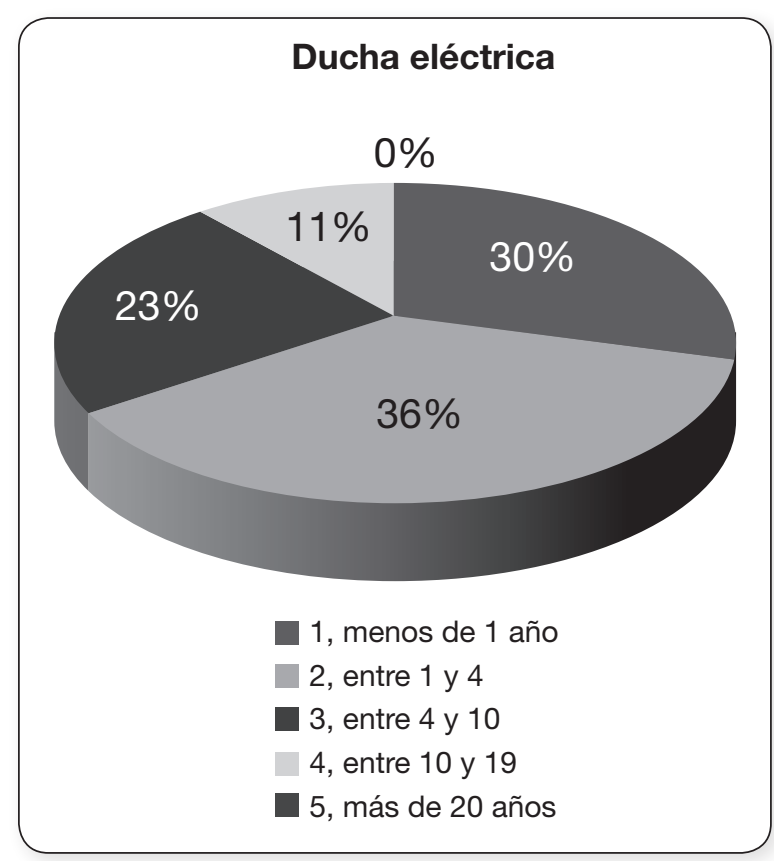

Gráfico 7. Antigüedad promedio de las duchas eléctricas. Fuente: Elaboración propia a partir de la información de la encuesta.

\section{b. Técnicos}

- Reducción del máximo consumo de la mañana, en el cual las duchas y calentadores eléctricos representan un 26\% 
del consumo; y un 10\% en el máximo consumo de la noche, esta reducción ayudará a mejorar el factor de carga del sistema.

\section{c. Ambientales:}

- Reducción de los gases de efecto invernadero ya que se requiere menor producción de energía eléctrica, además si se coloca calentadores termo-solares en vez de sistemas que usan GLP se reduce las emisiones debidas a la combustión.

- Cumplimiento con regulaciones internas de la CENTROSUR sobre gestión ambiental.

\section{d. Marketing, imagen corporativa}

El uso de energías renovables da al cliente una imagen de compromiso con la situación medioambiental, mejorando la imagen corporativa de la empresa.

\subsubsection{Formas de financiamiento}

Esta línea de acción puede recibir financiamiento por parte de organismos internacionales y fundaciones ${ }^{8}$. El Estado debería asumir la responsabilidad de estos proyectos brindando financiamiento y eliminando aranceles a la importación de estos equipos. La alternativa para masificar el uso de calentadores solares sería una campaña en la cual la CENTROSUR podría vender los calentadores y realizar el cobro mediante planillas; esto se puede apoyar con un bono por reducción de consumo, por ejemplo si un usuario sustituye una ducha por un sistema termo-solar y reduce su consumo en un $10 \%$ y mantiene esta reducción por un periodo, se le podría descontar un porcentaje de su deuda por la compra del equipo solar.

\subsubsection{Etapas de implementación}

a. Compromiso con socios estratégicos que suministren los equipos y financiamiento para la campaña.

b. Elaboración de proyectos piloto que adapten la tecnología a nuestro medio.

c. Difusión publicitaria de la campaña de sustitución de calentadores de agua.

d. Establecimiento de acuerdos comerciales con los clientes.

e. Sustitución de los equipos térmico-solares.

\subsubsection{Indicadores}

- Cantidad de duchas promedio por cliente.

- Cantidad de calentadores solares por cliente.

Existe una investigación realizada en el año 2007 (Vanegas, 2007) sobre aplicaciones térmicas en solares para la ciudad de Cuenca, a continuación se incluyen los resúmenes de este trabajo.

\subsection{Retraso de inversiones en expansión del Sistema de Sub-transmisión y Distribución}

Anualmente el consumo del sistema CENTROSUR crece a un ritmo de aproximadamente un 6\%; paralelamente, para satisfacer los requerimientos de los clientes es necesario que se realicen inversiones en subtransmisión y distribución para ampliar la capacidad de las redes. Si se aplica un Plan de Gestión de la Demanda Eléctrica es posible retardar estas inversiones utilizando las redes actuales de manera más eficiente. Se determinó que si se aplica un Plan de la Gestión de la Demanda es posible reducir un 9,4\% de la demanda en la hora 
pico de todo el sistema de la CENTROSUR, esto quiere decir que se puede retrasar en alrededor de un año las inversiones en ampliación de la capacidad de la red.

La ampliación o mejoras de las redes y subestaciones de la CENTROSUR se realizan siguiendo algunos parámetros:

a. Atender la demanda de los clientes.

b. Mantener los niveles de confiabilidad del sistema CENTROSUR.

c. Mantener los niveles de calidad de la energía exigidos por las regulaciones.

Para realizar el presente análisis se considerará que las inversiones en redes y subestaciones únicamente se basan en la demanda máxima; es decir, las ampliaciones de la red sirven para satisfacer la máxima demanda, sin considerar la calidad de la energía o la confiabilidad del sistema eléctrico.

\subsection{Participación de la CENTROSUR en el mercado de bonos de carbono}

De acuerdo con el protocolo de Kioto, firmado en 1997, los Estados se comprometieron a reducir sus emisiones de carbono al 95\% de los niveles de emisión global en 1990. Como medio para motivar esta reducción se plantearon mecanismos 'laterales' para reducir las emisiones de gases de efecto invernadero (GEI) uno de estos mecanismos son los llamados bonos 'verdes' o bonos de carbono, que consisten en asignar cupos de emisiones de gases a los países participantes. Si un país ha excedido su cupo de emisión puede comprar esta capacidad de emisión a países con menor producción de gases, creándose un mercado de 'cupos' para emitir gases contaminantes. Este mercado tiene un gran dinamismo movilizando un capital importante.

La CENTROSUR puede participar en este mercado 'vendiendo su reducción' de gases de efecto invernadero, para lo cual deberá validar sus acciones y ofertar estos bonos en el mercado.

\subsubsection{Objetivos}

a. Validar y certificar la reducción de las emisiones de gases de efecto invernadero.

b. Vender los bonos de reducción de gases de efecto invernadero.

\subsubsection{Actores}

\section{a. Primarios}

- Empresa Eléctrica Regional Centro Sur, compradores de bonos de carbono.

\section{b. Secundarios:}

- Los clientes de la Empresa Eléctrica Regional Centro Sur.

\subsubsection{Barreras para la participación en el mercado de bonos de carbono}

La principal dificultad radica en la justificación de la reducción de emisiones. El tratado de Kioto indica que se puede acceder y vender los cupos de carbono si la medida adoptada para reducir las emisiones no era obligatoria por alguna ley o reglamento vigente en el país.

8 La Fundación Ecológica Rikcharina instaló un sistema termo-solar y fotovoltaico en la comunidad Aguarongo que queda en el bosque del mismo nombre en la provincia del Azuay. Dirección en internet: http://www.rikcharina.org/paginas/aguarongo.htm 


\subsubsection{Beneficios}

Ingresar al mercado de bonos de emisión de gases de efecto invernadero permitiría a la CENTROSUR integrarse a un mercado global que exige altos niveles de competitividad y mejora constante de procesos para reducir las emisiones de gases, los beneficios son:

\section{a. Económicos}

- La venta de bonos de carbono representaría un ingreso adicional para la CENTROSUR e iniciaría un nuevo giro de negocio ya que la empresa no solo vendería energía sino que participaría en un mercado de nuevos servicios.

- Creación de fuentes de trabajo en torno a las negociaciones de los bonos.

\section{b. Técnicos}

La empresa lideraría el mercado local y podría capacitar a otras industrias del medio para que participen en este mercado.

\section{c. Ambientales}

Reducción de los gases de efecto invernadero, ya que se obtendrán ganancias por cada $\mathrm{kW}$ disminuido?.

\section{d. Marketing, imagen corporativa}

La CENTROSUR se presentará como pionera en el sector eléctrico del país en intervenir en este mercado global.

\subsubsection{Formas de financiamiento}

El financiamiento de este proyecto sería cubierto por sí mismo ya que al iniciar las negociaciones, el ahorro producido por este plan de Gestión de Consumo de Energía será recompensado económicamente en forma de apoyo para otros proyectos de ahorro y eficiencia eléctrica.

\subsubsection{Etapas de implementación}

a. Capacitación y difusión del Acuerdo de Kioto.

b. Determinación de la cantidad de gases reducidos mediante el plan de gestión de la demanda.

c. Compromiso con socios estratégicos nacionales e internacionales para recibir asesoría en el proyecto.

d. Certificación sobre los procesos de reducción de emisiones de GEI, (por ejemplo: Certificaciones Gold Estándar) ${ }^{10}$.

e. Establecimiento de acuerdos comerciales agentes del mercado de carbono.

\subsubsection{Indicadores}

- Cantidad de bonos vendidos.

- Ingresos económicos por la venta de bonos.

\section{Certificación Gold Standar}

Gold standar es un esquema de certificación $\mathrm{y}$ verificación ambiental que permite identificar y determinar si los proyectos de reducción de emisiones de GEI son sostenibles en el tiempo y cumplen con las metas planteadas en los acuerdos. Estas certificaciones solo se dan a proyectos de energías renovables y eficiencia energética.

9 Como nota interesante: en España se denominó 'negavatio' a la energía reducida mediante los proyectos de eficiencia energética.

10 La dirección electrónica de Gold Standard es http://www.cdmgoldstandard.org/index.php, en la cual existe información sobre los criterios para la certificación. 
A continuación se enumera los proyectos que Gold Standar certifica:

\subsection{Energía renovable}

Energía eólica, hidroeléctrica, solar-térmica, solar-fotovoltaica, biomasa, biogás, geotérmica.

\subsection{Eficiencia energética}

Los proyectos de gestión de consumo de energía (DSM o PGD) y programas de eficiencia energética, los programas que busquen una disminución del consumo energético y contribuyan a reducir el consumo de energías primarias fósiles aplican a la certificación Gold Standar.

Los programas de eficiencia en los sectores industrial, residencial, comercial y transporte son aplicables para la certificación Gold Standar.

Los diseñadores de proyectos y programas deben contactar con la secretaría de Gold Standar para confirmar si su proyecto es elegible, el criterio de selección no es estático y en muchos casos se aplican reglas de selección.

Cuando una empresa desee vender bonos de carbono bajo el Mecanismo de Desarrollo Limpio (MDL) tiene que cumplir el requisito de la adicionalidad; es decir, demostrar que no habría incorporado nuevas tecnologías si no fuera por este mecanismo flexible, de esta manera no habría realizado el proyecto por otra razón que no sea participar en el mercado de bonos. Una vez formulado el proyecto una entidad operacional independiente (como Gold Standar), acreditada ante la Junta Ejecutiva del Protocolo, se estima la reducción de emisiones que el proyecto en funcionamiento puede producir. Luego, la Junta Ejecutiva del Mecanismo de Desarrollo Limpio emite el bono de carbono, pudiéndose vender en el mercado. Una vez llevado a cabo el proyecto, una segun$\mathrm{da}$ entidad operacional independiente verifica y cuantifica la reducción efectiva de emisiones.

Desde el punto vista de la CENTROSUR, los programas de energía solar térmica y eficiencia energética son aplicables para la certificación Gold Standar, y su posterior participación en los mercados de carbono.

\section{Análisis económico del impacto de las medidas del Plan de gestión del consumo residencial}

Con este marco conceptual se planean las acciones que contempla el plan de gestión de demanda, las propuestas que se presentan a continuación han sido contrastadas con la información obtenida en las encuestas realizadas a los clientes.

Las propuestas que requieren una inversión son las siguientes:

- Campaña de ahorro de energía: reducción del uso de equipos en las horas de mayor consumo, eliminar el consumo stand-by.

- Campaña de eficiencia energética: sustitución de focos incandescentes por lámparas fluorescentes compactas, cambio de equipos de refrigeración por equipos eficientes.

- Sustitución de calentadores eléctricos de agua por calentadores solares.

A continuación se define cada una de ellas.

\subsection{Campaña de ahorro de energía}

Consideramos ahorro energético a la disminución en el consumo innecesario de energía 'apagando las luces', es decir dejando de realizar un consumo. Esta reducción se enfocará 
sobre todo durante la hora de máximo consumo que para el sector residencial de Cuenca se da a las $20 \mathrm{~h} 00$.

Se propone reducir el consumo stand by en un $50 \%$; esto se logra conectando los artefactos eléctricos a regletas con interruptor, pues impide que los equipos consuman energía para mantener relojes internos $\mathrm{u}$ otros dispositivos en modo de 'espera'.

Con esta medida se lograría reducir 8,855 MWh/mes y 290,9415 kW en la hora de máximo consumo de la noche.

Otra alternativa es la reducción del consumo en un equipo presente en todo hogar: el televisor. En la investigación se determino las siguientes particularidades:

- En el cantón Cuenca existen aproximadamente 4 equipos de televisión por cada domicilio.

- Durante las horas de máximo consumo casi todos estos equipos se encienden.

- El uso de este equipo representa cerca del $80 \%$ del grupo radio-tv.

Se propone realizar una campaña de uso eficiente de energía en la cual se reduzca el uso de un equipo de tv en cada hogar durante la hora pico, esto significaría una disminución de 3,738 MWh/mes, y $122,8331 \mathrm{KW}$ durante la hora de máximo consumo.

\subsection{Campaña de eficiencia energética}

La eficiencia energética centra sus medidas al cambio o sustitución de equipos por similares pero de mejor eficiencia. En esta línea se plantea la sustitución de focos incandescentes por lámparas fluorescentes compactas; esta campaña se está llevando a cabo en la actualidad en el país con buenos resultados.
En promedio las lámparas fluorescentes compactas (LFC) consumen una quinta parte de la energía entregada a una lámpara incandescente iluminado igual. Se presenta un problema con este tipo de lámparas ya que poseen un sistema de conversión basado en un puente rectificador de diodos, como es conocido este tipo de fuentes producen la degradación de la forma de onda y otras distorsiones, por tanto afectan a la calidad de la energía suministrada por la empresa comercializadora y reducen la eficiencia del sistema en general (Eguíluz et al., 2006), aumentando las perdidas en el transporte.

Con esta medida se logra una disminución de 43,335 MWh/mes y una reducción de 7,222 MW en la hora de máximo consumo.

Otra alternativa es el cambio de equipos de refrigeración por equipos más eficientes. En las encuestas se observa que un $5 \%$ de los usuarios planea comprar una refrigeradora o congelador en los próximos meses; si se realizara un cambio de alrededor de 12.000 refrigeradoras tendríamos una disminución de $55.374 \mathrm{MWh} /$ mes ó 1.819,3 MW en la hora de máximo consumo y a lo largo de cada hora del día.

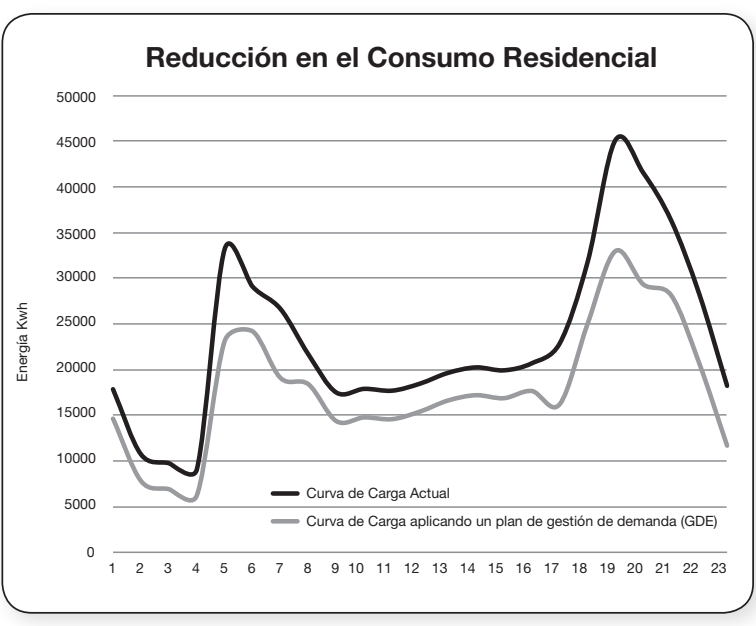

Gráfico 8. Relación entre las curvas de Carga. 


\subsection{Sustitución de calentadores eléctricos de agua por calentadores solares}

Al analizar la conveniencia de utilizar calentadores solares para los domicilios existe un gran potencial; al realizar un cambio de calentadores eléctricos por calefón que usa GLP en un 10\% de los usuarios (alrededor de 20.000 clientes). Se lograría disminuir 8.121,44 MWh/ mes y 1,072 MWh en la hora de máximo consumo en la mañana y en la noche.

La otra propuesta consiste en reemplazar un $1 \%$ de todos los calentadores eléctricos por equipos termo-solares, esta medida alcanzaría aproximadamente 2.000 equipos, en términos de energía ahorrada esta disminución representa mensualmente $8.121 \mathrm{kWh}$.

La Tabla 1: Energía Reducida aplicando el Plan de Gestión de Consumo presenta la mejora en el factor de carga en un 2\% al aplicar el plan de gestión de consumo y se reduce un $26 \%$ del consumo de energía para el sector residencial.

La Tabla 2: Energía Reducida en cada Grupo de Consumo indica el porcentaje de reducción de cada grupo de electrodomésticos.

Energía Reducida kWh/mes

\begin{tabular}{|c|c|c|c|c|c|}
\hline Consumo & Factor de Carga & $\begin{array}{c}\text { Consumo } \\
\text { de Energía }\end{array}$ & $\begin{array}{c}\text { En la hora } \\
\text { de máximo } \\
\text { consumo noche }\end{array}$ & $\begin{array}{c}\text { En la hora } \\
\text { de máximo } \\
\text { consumo de } \\
\text { la mañana }\end{array}$ & Unidad \\
\hline $\begin{array}{c}\text { Actual } \\
\text { Aplicando } \\
\text { el PGC }\end{array}$ & 0,52 & 544638,71 & 45117,42 & 33199,28 & $\mathrm{kWh} / \mathrm{mes}$ \\
$\begin{array}{c}\text { Diferencia } \\
\text { Variación }\end{array}$ & 0,53 & 404762,88 & 32967,60 & 24181,70 & $\mathrm{kWh} / \mathrm{mes}$ \\
\hline
\end{tabular}

Tabla 1. Energía Reducida aplicando el Plan de Gestión de Consumo

\section{Reducción en el consumo. KWH/mes}

\begin{tabular}{|c|c|c|c|}
\hline & Consumo & Reducción & $\%$ \\
\hline Iluminación & 108031,21 & 43335,54 & $40,11 \%$ \\
\hline Preparacion Alimentos & 30370,94 & 0,00 & \\
\hline Radio Tv Entretenimiento & 62419,42 & 3738,73 & $5,99 \%$ \\
\hline Aseo Limpieza Varios & 50686,91 & 0,00 & \\
\hline Computación & 97482,05 & 4815,96 & $4,94 \%$ \\
\hline Calentamiento de Agua & 21609,70 & 8121,45 & $37,58 \%$ \\
\hline Refrigeración & 155557,38 & 55374,80 & $35,60 \%$ \\
\hline Stand By & 18481,11 & 8855,53 & $47,92 \%$ \\
\hline Totales & 544638,71 & 139875,83 & $25,68 \%$ \\
\hline
\end{tabular}

Tabla 2. Energía Reducida en cada Grupo de Consumo 


\section{Beneficio económico de las medidas de ahorro y eficiencia energética, a nivel de consumidor y empresa}

\subsection{Pérdidas de energía}

Con base en los datos de la CENTROSUR conocemos que las pérdidas técnicas promedio durante el año 2008 en el sistema equivalen aproximadamente al 5,83\% ${ }^{11}$ de la energía disponible para la comercializadora. El precio promedio de compra de la energía para junio de 2008 fue de US $\$ 5,11 / \mathrm{kWh}^{12}$.

El ahorro económico para la CENTROSUR por la reducción de las pérdidas de energía para el sector residencial del cantón Cuenca asciende aproximadamente US \$ 6.606,12 mensuales.

\subsection{Emisiones de $\mathrm{CO}_{2}$}

Las emisiones de carbono debido a la producción de electricidad, usando diversas tecnologías y distintas fuentes equivalen aproximadamente a $1,535 \mathrm{lb} \mathrm{CO} 2 / \mathrm{kWh}^{13}$. En el año 2008, un $36,28 \%$ de la generación se realizó con generación térmica.

Los bonos de carbono pueden tener un precio promedio de entre US $\$ 20$ y $30^{14}$ por Tonelada de $\mathrm{CO}_{2}$ desplazado.

Si se reemplaza calentadores que queman GLP por sistemas termo-solares se tiene una reducción en las emisiones de $\mathrm{CO}_{2}$ debido a la disminución en la cantidad de GLP combustionado; la relación de emisiones de $\mathrm{CO}_{2}$ por cada $\mathrm{kg}$ de hidrocarburo, se presenta a continuación.

\begin{tabular}{|c|c|c|c|c|}
\hline \multicolumn{5}{|c|}{ Energía Mensual Consumida para el sector residencial del cantón Cuenca } \\
\hline Actual & $533.920,69$ & $\mathrm{kWh} / \mathrm{mes}$ & & \\
\hline Aplicando el GDE & $411.764,14$ & $\mathrm{kWh} / \mathrm{mes}$ & & \\
\hline \multicolumn{3}{|c|}{ Porcentaje de Pérdidas técnicas en el sistema Centrosur: } & $5,83 \%$ & \\
\hline \multicolumn{3}{|c|}{ Precio Promedio de Energía: } & 5,11 & Cent USD/kWh \\
\hline \multicolumn{5}{|c|}{ Energía Mensual requerida para el sector residencial del cantón Cuenca } \\
\hline & $\mathrm{kWh} / \mathrm{mes}$ & \multicolumn{2}{|c|}{ Estimado de pago por la energía comprada } & \\
\hline Sin aplicar el GDE & $565.048,27$ & \multicolumn{2}{|c|}{$\$ 28.873,97$} & Se calculó la \\
\hline Aplicando el GDE & $435.769,99$ & \multicolumn{2}{|c|}{$\$ 22.267,85$} & $\begin{array}{l}\text { energía requerida, } \\
\text { sumando la energía }\end{array}$ \\
\hline Diferencia: & $129.278,28$ & \multicolumn{2}{|c|}{$\$ 6.606,12$} & consumida más las \\
\hline \multicolumn{2}{|c|}{$\begin{array}{l}\text { "Ahorro Producido por el PGD } \\
\text { en las pérdidas de energía" }\end{array}$} & \multicolumn{2}{|c|}{$\$ 6.606,12$ USD } & pérdidas técnicas \\
\hline
\end{tabular}

Tabla 3. Análisis de Pérdidas de Energía

11 Este valor promedio se obtuvo de los informes enviados al CENACE por parte de la CENTROSUR.

12 Obtenido del boletín "Estadística del sector eléctrico Ecuatoriano primer semestre del año 2008”, publicado por el CENACE.

13 Datos de EPA (Enviroment Protection Agency) 2006.

14 El Mecanismo de Desarrollo Limpio en Ecuador: Un diagnóstico rápido de los retos y oportunidades en el Mercado de Carbono; Oficina Ecuatoriana de Promoción del MDL - CORDELIM; Quito, 2006. 


\begin{tabular}{|c|c|c|c|}
\hline \multicolumn{4}{|c|}{ Participación de las Tecnologías en Generación (Promedio País) } \\
\hline Tipo de Generación & $\begin{array}{l}\text { Porcentaje de } \\
\text { Participación }\end{array}$ & & \\
\hline Térmica & $48,00 \%$ & \multirow{2}{*}{\multicolumn{2}{|c|}{$\begin{array}{l}\text { Nota: históricamente el promedio de generación } \\
\text { termo-eléctrico se acerca al } 48 \% \text { de la produc- } \\
\text { ción energética anual, el año } 2008 \text { fue un buen } \\
\text { año en términos hidrológicos para el país. }\end{array}$}} \\
\hline Hidráulica & $52,00 \%$ & & \\
\hline \multicolumn{4}{|c|}{ Energía Mensual requerida para el sector residencial del cantón Cuenca } \\
\hline Actual (sin aplicar la GDE) & 565048,2661 & \multicolumn{2}{|c|}{$\mathrm{kWh}$} \\
\hline Aplicando la GDE & 435769,9865 & \multicolumn{2}{|r|}{ kWh } \\
\hline \multicolumn{4}{|c|}{ Energía Mensual requerida para el sector residencial por tipo de tecnología del Generador } \\
\hline & Térmica & Hidráulica & \\
\hline Sin aplicar el PGD & 271223,1677 & 293825,0984 & kWh \\
\hline Aplicando el PGD & 209169,5935 & 226600,393 & $\mathrm{kWh}$ \\
\hline Reducción & 62053,57421 & 67224,70539 & $\mathrm{kWh}$ \\
\hline \multicolumn{4}{|c|}{ Reducción de emisiones de GEI por generadoras } \\
\hline $\begin{array}{l}\text { Cantidad de } \mathrm{CO}_{2} \text { produ- } \\
\text { cido por cada } \mathrm{kWh}\end{array}$ & 1,53 & lb CO2/kWh & \\
\hline \multirow{2}{*}{$\begin{array}{l}\text { Cantidad de } \mathrm{CO}_{2} \text { reducido por la } \\
\text { aplicación del PGD en un mes }\end{array}$} & 94941,96854 & $\mathrm{lb} \mathrm{CO}_{2}$ & \multirow{2}{*}{$\begin{array}{l}\text { Multiplicando la ener- } \\
\text { gía reducida por } 1,53 \\
\text { lib } \mathrm{CO}_{2} \text { por } \mathrm{kWh}\end{array}$} \\
\hline & 43,0649468 & Ton $\mathrm{CO}_{2}$ & \\
\hline \multicolumn{4}{|c|}{ Reducción de emisiones de GEI por reducción de uso de GLP en calentamiento de agua } \\
\hline Factor & Valor & Unidades & Nota \\
\hline $\begin{array}{l}\text { Cantidad de } \mathrm{CO}_{2} \text { produci- } \\
\text { do por cada kg de GLP }\end{array}$ & 3 & $\mathrm{~kg} \mathrm{CO} / \mathrm{kg}$ de GLP & Con datos de EPA \\
\hline $\begin{array}{l}\text { Cantidad de Calenta- } \\
\text { dores sustituidos }\end{array}$ & 2000 & Unidades & $\begin{array}{l}\text { Sustituyendo un } 1 \% \text { de los } \\
\text { calentadores a GLP existentes } \\
\text { por equipos termosolares. }\end{array}$ \\
\hline $\begin{array}{l}\text { Kg de GLP Desplazado } \\
\text { por cada calentador }\end{array}$ & 30 & Kg de GLP & $\begin{array}{l}\text { Al reducir el uso de } 2 \text { cilíndros } \\
\text { de } 15 \mathrm{~kg} \text { cada mes }\end{array}$ \\
\hline $\begin{array}{l}\text { Cantidad de } \mathrm{CO}_{2} \text { reducido } \\
\text { por el reemplazo de calenta- } \\
\text { dores que consumen GLP por } \\
\text { calentadores termo-solares }\end{array}$ & 180 & Ton $\mathrm{CO}_{2}$ & $\begin{array}{l}\text { Multiplicado: "Cantidad de } \\
\text { CO2 producido por cada Kg } \\
\text { de GLP" por "Cantidad de Ca- } \\
\text { lentadores sustituidos" y por } \\
\text { "kg de GLP Desplazado } \\
\text { por cada calentador" }\end{array}$ \\
\hline \multicolumn{4}{|c|}{ Volumen de CO2 desplazado por el PGD } \\
\hline \multicolumn{2}{|c|}{ Reducción de Emisiones en Generación de E. Eléctrica } & 43,06 & Ton $\mathrm{CO}_{2}$ \\
\hline \multicolumn{2}{|c|}{ Reducción de Emisiones en Combustión de GLP } & 180 & Ton $\mathrm{CO}_{2}$ \\
\hline \multicolumn{2}{|c|}{ Total de Emisiones evitadas aplicando el PGD } & 223,06 & Ton $\mathrm{CO}_{2}$ \\
\hline \multicolumn{4}{|c|}{ Ingresos por la venta de Bonos de Carbono } \\
\hline \multicolumn{2}{|c|}{ Precio de una Ton de $\mathrm{CO}_{2}$ en el mercado. } & 25 & $\mathrm{USD} / \mathrm{Ton} \mathrm{CO}_{2}$ \\
\hline \multicolumn{2}{|c|}{ Ingresos por la venta de Bonos de Carbono } & $\$ 5.576,62$ & USD \\
\hline
\end{tabular}

Tabla 4. Ingresos por la venta de bonos de carbono 
Cada calentador de GLP consume alrededor de dos cilindros de $15 \mathrm{~kg}$ al mes. El GLP es un hidrocarburo de alta concentración de carbono, lo equiparamos a las emisiones producidas por el propano que emite $3 \mathrm{~kg}$ de $\mathrm{CO}_{2}$ por cada $\mathrm{kg}$ de combustible usado.

Como muestra la Tabla 4, al reemplazar 2.000 calentadores de agua en que queman GLP por calentadores solares se evita producir 212,55 ton de $\mathrm{CO} 2$, si se participa en los mercados de carbono esta reducción puede representar un ingreso económico de US \$ 5.313,75 mensuales.

\begin{tabular}{|c|c|c|}
\hline Fórmula & $\begin{array}{c}\mathrm{Kg} \text { de } \mathrm{CO}_{2} \\
\text { de salida }\end{array}$ & Nombre \\
\hline $\mathrm{C}_{7} \mathrm{H}_{16}$ & $3,08 \mathrm{CO}_{2}$ & Heptano \\
$\mathrm{C}_{3} \mathrm{H}_{8}$ & $3 \mathrm{CO}_{2}$ & Propano \\
$\mathrm{CH}_{3} \mathrm{OH}$ & $1,375 \mathrm{CO}_{2}$ & $\begin{array}{c}\text { Alcohol } \\
\text { Metílico }\end{array}$ \\
$\mathrm{C}_{2} \mathrm{H}_{5} \mathrm{OH}$ & $1,91 \mathrm{CO}_{2}$ & Alcohol Etílico \\
\hline
\end{tabular}

Tabla 5. Niveles de emisión de $\mathrm{CO}_{2}$ de varios combustibles.

\subsection{Cargos por transmisión ${ }^{15}$}

En la estructura de costos de la energía las empresas distribuidoras deben cancelar un rubro al sistema de transmisión, este valor es llamado 'cargos por transmisión' y se compone de dos valores:

\subsubsection{Tarifa variable de transporte en el mercado de contratos}

Es una función de la cantidad de energía comprada por la comercializadora y la distancia a la que se encuentran las unidades de ge- neración, para este cálculo interviene al factor nodal.

\subsubsection{Tarifa fija por transporte en transmisión}

Es calculada a partir de la máxima demanda del sistema de una empresa comercializadora multiplicada por un valor fijo para todo el país, como se indica en la Tabla 6, en la regulación se indica que el valor fijo es de US $\$ 1,56 / \mathrm{kW}$.

$$
\text { TFTT }=\text { máx.dem. } * \text { Val.fijo }
$$

Ecuación 3. Cálculo de Tarifa fija por transporte en transmisión.

Donde:

TFTT: Tarifa fija por transporte en transmisión.

Máx.dem: Máximo valor de demanda en el periodo de un mes.

Val. Fijo: Constante de precio, expresado en USD $/ \mathrm{kW}$.

Aplicando la Gestión de Demanda Eléctrica en la hora pico es posible reducir 12.197,231 $\mathrm{kW}$. que equivale aproximadamente a una reducción del 9,4\%, con lo cual la máxima demanda disminuye y consecuentemente el monto de la TFTT es reducido en alrededor de US \$ 19.027,68 mensuales.

\subsection{Retraso de inversiones en expansión del Sistema de Subtransmisión y Distribución}

Anualmente el consumo del sistema CENTROSUR crece a un ritmo de aproximadamente un $6 \%$. Paralelamente para satisfacer los requerimientos de los clientes es necesario

15 Citado de: Regulación no. CONELEC - 014/99 administración técnica y operativa del sistema de transmisión, Publicada por el CONELEC, en vigencia desde 11 de noviembre de 1999. 


\begin{tabular}{|c|c|c|c|c|}
\hline Unidad & Cantidad & Descripción & Precio unitario & Total parcial \\
\hline \multicolumn{5}{|c|}{ CONSUMO ACTUAL } \\
\hline $\mathrm{kW}$ & 129722,292 & Tarifa fija por transporte en transmisión & $\$ 1,56$ & $\$ 202.366,78$ \\
\hline \multicolumn{5}{|c|}{ CONSUMO ESPERADO APLICANDO LA GDE } \\
\hline \multicolumn{5}{|c|}{ Reducción de 12197,231kW en la hora pico } \\
\hline \multirow[t]{2}{*}{$\mathrm{kW}$} & 117525,0606 & Tarifa fija por transporte en transmisión & $\$ 1,56$ & $\$ 183.339,09$ \\
\hline & & Diferencia en la tarifa fija por transporte & & $\$ 19.027,68$ \\
\hline
\end{tabular}

Tabla 6. Reducción en la Tarifa fija por transporte en transmisión, aplicando la GDE, cálculo realizado con valores del mes de enero de 2009.

que se realicen inversiones en subtransmisión y distribución para ampliar la capacidad de las redes. Si se aplica un Plan de Gestión de la Demanda Eléctrica es posible retardar estas inversiones utilizando las redes actuales de manera más eficiente. Se determinó que si se aplica un Plan de la Gestión de la Demanda es posible reducir un 9,4\% de la demanda en la hora pico de todo el sistema de la CENTROSUR, esto quiere decir que se puede retrasar en alrededor de un año las inversiones en ampliación de la capacidad de la red.

La ampliación o mejoras de las redes y subestaciones de la CENTROSUR se realizan siguiendo algunos parámetros:

- Atender la demanda de los clientes.

- Mantener los niveles de confiabilidad del sistema CENTROSUR.

- Mantener los niveles de calidad de la energía exigidos por las regulaciones.

Para realizar el presente análisis se considerará que las inversiones en redes y subestaciones únicamente se basan en la demanda máxima, es decir las ampliaciones de la red sirven para satisfacer la máxima demanda, sin considerar la calidad de la energía o la confiabilidad del sistema eléctrico.
Para la presente investigación se calculará únicamente los valores de inversiones en redes de distribución y subestaciones, esto se debe a que es muy dificil establecer cuáles son las inversiones que se realizan para el sector residencial; más aún, la mayor parte de las obras de ampliación de redes se realizan en los sectores suburbanos o en la periferia, el análisis, por lo tanto se complica; para los fines de la presente tesis se elaborará el análisis hasta las redes de distribución y subestaciones.

La Tabla 7 presenta los activos y anualidades de la CENTROSUR, como se explico antes, sólo se considerará los valores de las redes de subtransmisión y las subestaciones.

Para el periodo 2009, la CENTROSUR deberá gastar US $\$ 810.487,60$ en mantener estos activos en funcionamiento, por lo cual este valor no puede ser descontado de las inversiones y no se lo considerará en los cálculos.

Según valores que muestra la Tabla 8, para el presente año la CENTROSUR tiene proyectos de ampliaciones de las redes y subestaciones aproximadamente por US \$1'756.684,00 de los cuales US $\$ 871.860,00$ serán cubiertos por la CENTROSUR. A este valor se le descuenta las anualidades tenemos US \$946.196,40; de los cuales $\$ 469.509,87$ serán cubiertos por la CENTROSUR. 


\begin{tabular}{|c|c|c|c|}
\hline \multicolumn{4}{|c|}{ Activos en servicio y anualidades } \\
\hline & Activos año 2009 & Vida Útil & Anualidad \\
\hline & US\$ & Años & US\$ \\
\hline Lineas de Subtransmisión & $\$ 13.915 .481,06$ & 45 & $\$ 309.232,91$ \\
\hline S/E de Distribución & $\$ 15.037 .640,54$ & 30 & $\$ 501.254,68$ \\
\hline Alimentadores Primarios & $\$ 63.927 .213,25$ & 35 & $\$ 1.826 .491,81$ \\
\hline $\begin{array}{l}\text { Transformadores } \\
\text { de Distribución }\end{array}$ & $\$ 10.832 .230,24$ & 30 & $\$ 361.074,34$ \\
\hline Red Secundaria & $\$ 46.914 .014,29$ & 35 & $\$ 1.340 .400,41$ \\
\hline Alumbrado Público & $\$ 9.286 .702,07$ & 25 & $\$ 371.468,08$ \\
\hline $\begin{array}{l}\text { Instalaciones de Ser- } \\
\text { vicio al Cliente }\end{array}$ & $\$ 24.819 .808,28$ & 20 & $\$ 1.240 .990,41$ \\
\hline Instalaciones Generales & $\$ 21.001 .415,70$ & 10 & $\$ 2.100 .141,57$ \\
\hline TOTALES & $\$ 205.734 .505,43$ & & $\$ 8.051 .054,22$ \\
\hline \multicolumn{4}{|c|}{ Activos y anualidades de redes y sub-estaciones } \\
\hline & Activos año 2009 & Vida Útil & Anualidad \\
\hline & US\$ & Años & US\$ \\
\hline Lineas de Subtransmisión & \$ 13.915.481,06 & 45 & $\$ 309.232,91$ \\
\hline S/E de Distribución & $\$ 15.037 .640,54$ & 30 & $\$ 501.254,68$ \\
\hline TOTALES & $\$ 28.953 .121,60$ & & $\$ 810.487,60$ \\
\hline
\end{tabular}

Tabla 7. Activos y anualidades de la CENTROSUR para el año 2009

Se conoce que el sector residencial consume aproximadamente el 49\% de la energía suministrada por la CENTROSUR; esto representa una inversión cercana a US \$230.059,84 para el sector residencial, además el 69\% de los clientes del sector residencial de la CENTROSUR se encuentran en el cantón Cuenca equivale a US \$158.741,29 anuales que podrían ser retrasados al aplicar la gestión de la demanda eléctrica.
Aplicando esta metodología se aprecia que el Estado ecuatoriano puede ahorrarse US\$ 161.167,72 como muestra la Tabla 10.

El plan de gestión de consumo planteado considera una reducción de $122 \mathrm{MWh} /$ mes y $12 \mathrm{MW}$ en las horas de mayor consumo en la noche. 


\begin{tabular}{|c|c|c|c|}
\hline \multicolumn{4}{|c|}{ Inversiones previstas para el año 2009} \\
\hline Descripción & Presupuestado & Empresa & MEER \\
\hline SUBESTACIONES & $\$ 946.158,00$ & $\$ 510.417,00$ & $\$ 435.741,00$ \\
\hline LINEAS A 69 KV & $\$ 810.526,00$ & $\$ 361.263,00$ & $\$ 449.263,00$ \\
\hline Total & $\$ 1.756 .684,00$ & $\$ 871.680,00$ & $\$ 885.004,00$ \\
\hline Participación & & $49,62 \%$ & $50,38 \%$ \\
\hline \multicolumn{4}{|c|}{ Inversiones en mantenimiento de las redes y sub-estaciones. (Anualidades) } \\
\hline Descripción & Presupuestado & Empresa & MEER \\
\hline LINEAS A $69 \mathrm{KV}$ & $\$ 309.232,91$ & 153.444 & 155.789 \\
\hline SUBESTACIONES & $\$ 501.254,68$ & 248.726 & 252.528 \\
\hline Total & $\$ 810.487,60$ & $\$ 402.170,13$ & $\$ 408.317,47$ \\
\hline \multicolumn{4}{|c|}{ Inversiones previstas para el año 2009 , descontado Anualidades } \\
\hline Descripción & Presupuestado & Empresa & MEER \\
\hline LINEAS A $69 \mathrm{KV}$ & $\$ 636.925,09$ & 316.047 & 320.878 \\
\hline SUBESTACIONES & $\$ 309.271,32$ & 153.463 & 155.809 \\
\hline Total & $\$ 946.196,40$ & $\$ 469.509,87$ & $\$ 476.686,53$ \\
\hline \multicolumn{4}{|c|}{ Proporción de la participación del sector residencial en las inversiones. } \\
\hline & & \multicolumn{2}{|c|}{ Participación en el gasto } \\
\hline Sector de Consumo & Participación \% & Total & CENTROSUR \\
\hline Residencial & $49 \%$ & $\$ 463.636,24$ & $\$ 230.059,84$ \\
\hline Comercial & $9 \%$ & $\$ 85.157,68$ & $\$ 42.255,89$ \\
\hline Industrial & $27 \%$ & $\$ 255.473,03$ & $\$ 126.767,67$ \\
\hline Alumbrado Público & $9 \%$ & $\$ 85.157,68$ & $\$ 42.255,89$ \\
\hline Otros & $6 \%$ & $\$ 56.771,78$ & $\$ 28.170,59$ \\
\hline Total de inversiones. & & $\$ 946.196,40$ & $\$ 469.509,87$ \\
\hline \multicolumn{4}{|c|}{ Consumo del Sector Residencial del Cantón Cuenca con respecto al resto del sistema CENTROSUH } \\
\hline & Participación \% & \multicolumn{2}{|c|}{ Participación en el gasto } \\
\hline & & Total & CENTROSUR \\
\hline Cantón Cuenca & $69 \%$ & $\$ 319.909,00$ & $\$ 158.741,29$ \\
\hline Resto CENTROSUR & $31 \%$ & $\$ 143.727,23$ & $\$ 71.318,55$ \\
\hline \multicolumn{2}{|c|}{$\begin{array}{l}\text { Total de inversiones de la CENTROSUR para el sector } \\
\text { Residencial }\end{array}$} & $\$ 463.636,24$ & $\$ 230.059,84$ \\
\hline \multicolumn{3}{|c|}{$\begin{array}{l}\text { Ahorro en las inversiones de la CENTROSUR } \\
\text { para el sector Residencial del cantón Cuenca }\end{array}$} & $\$ 158.741,29$ \\
\hline \multicolumn{3}{|c|}{$\begin{array}{l}\text { Ahorro en las inversiones del Estado Ecuatoriano } \\
\text { para el sector Residencial del cantón Cuenca }\end{array}$} & $\$ 161.167,72$ \\
\hline
\end{tabular}

Tabla 8. Inversiones en redes y subestaciones previstas para el año 2009 


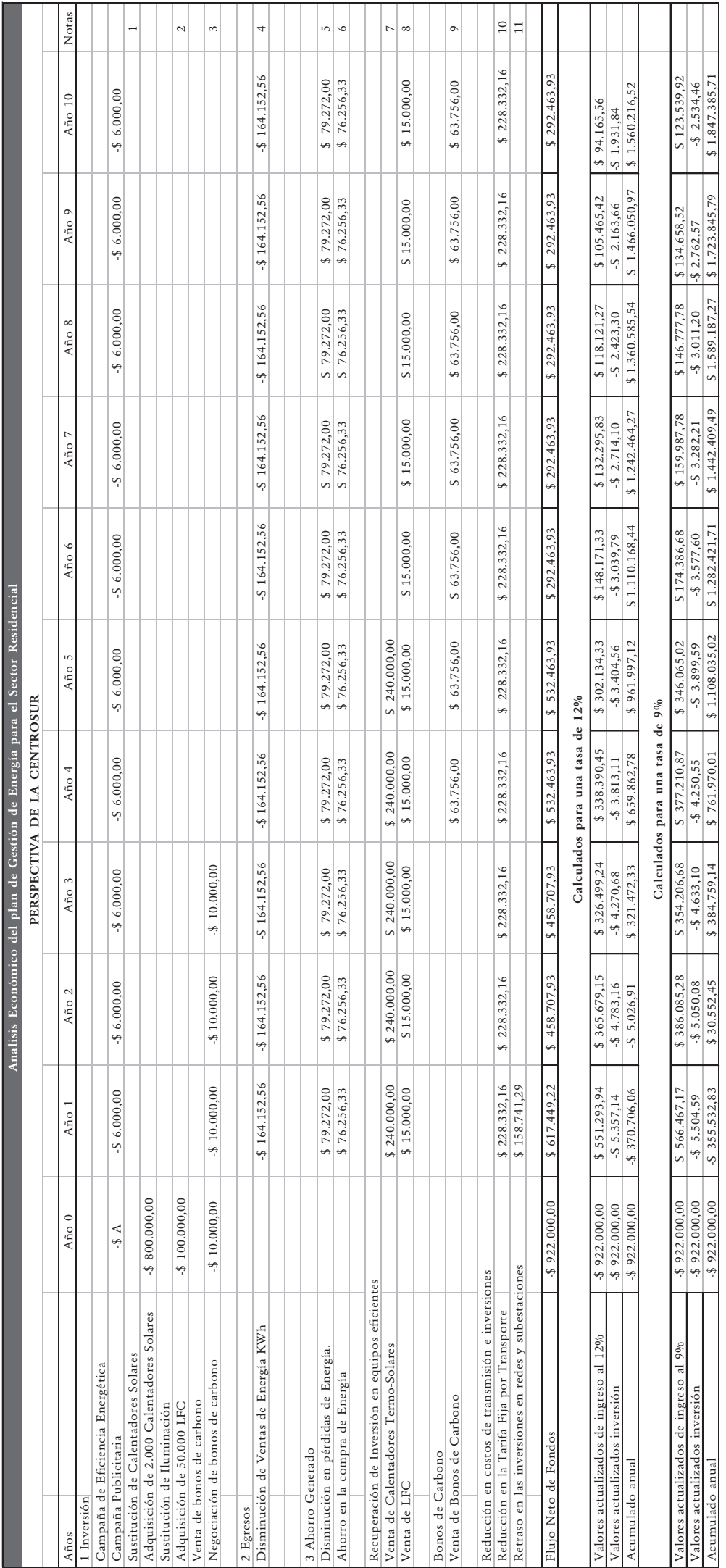
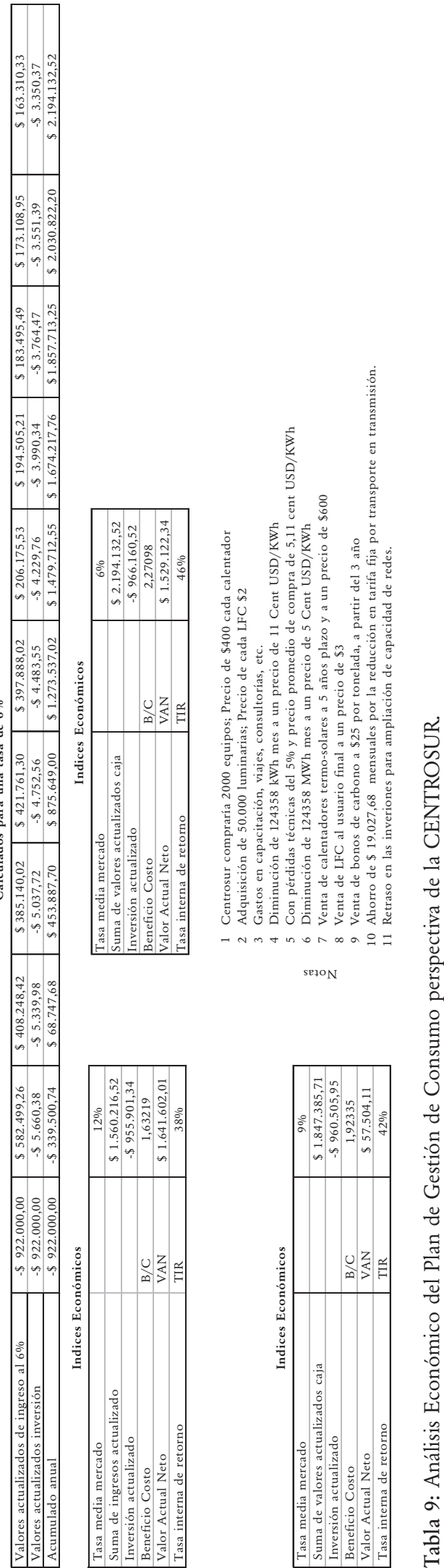


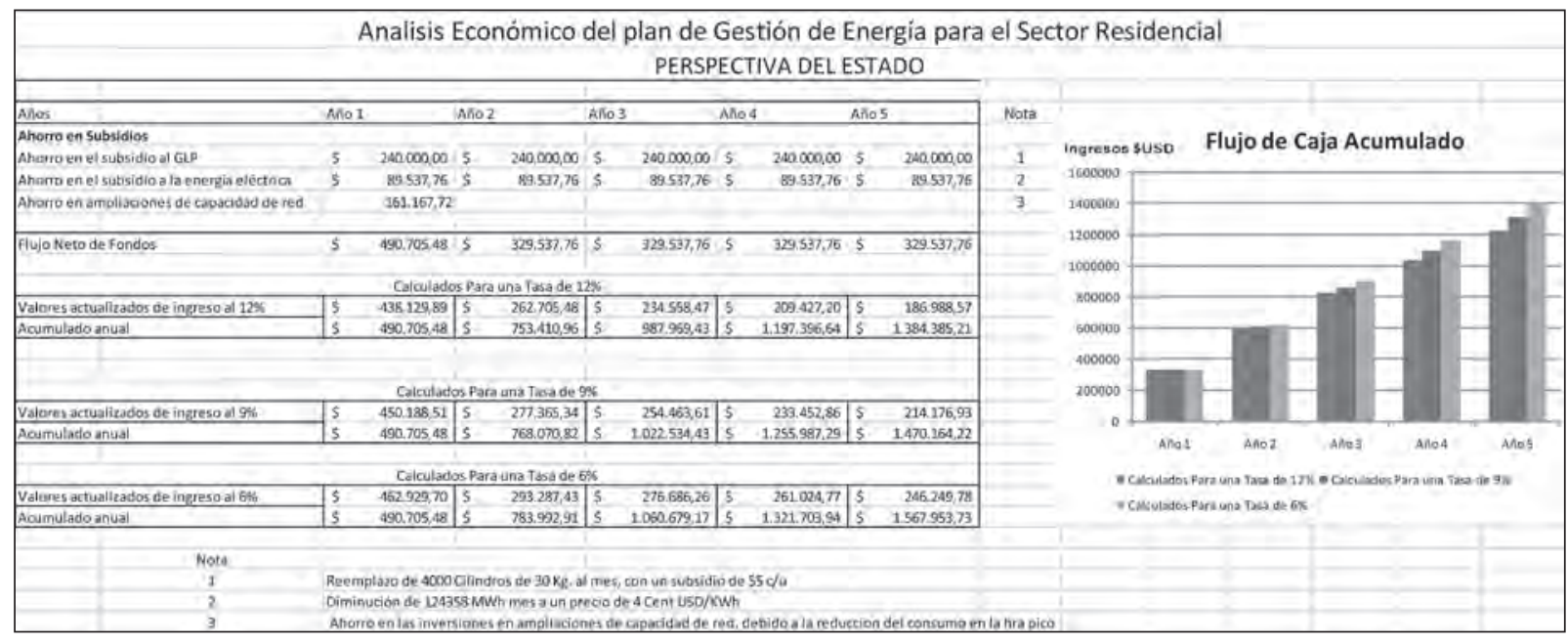

Tabla 10: Análisis Económico del Plan de Gestión de Consumo Residencial, desde el punto de vista del Estado.

\section{Conclusiones}

El análisis económico indica que la elaboración de planes de gestión de la demanda representa un gran beneficio económico para la empresa comercializadora ya que es posible reducir los egresos en los cargos por transmisión y se optimiza la gestión de recursos retrasando algunas inversiones que se realizarían para ampliar la capacidad de redes y subestaciones.

Durante la interacción con los encuestados se puso en relevancia la buena imagen que tiene la CENTROSUR ante sus clientes por su buen servicio, lo cual es beneficioso para realizar un proyecto que requiere inversión por parte de los clientes, ya que estos confian en las acciones y propuestas.

La CENTROSUR puede participar activamente en el mercado de los bonos de carbono, ofertando los cupos de emisión generados por la aplicación de las estrategias sugeridas en esta tesis.

\subsection{Curvas de Carga}

En las curvas de carga se observa dos periodos durante el día en los cuales el consumo de energía se incrementa estos son:

- 07h00 debido a la los grupos de iluminación, preparación de alimentos y calentamiento de agua.

- $20 \mathrm{~h} 00$ producido básicamente por el consumo de iluminación y radio / T.V.

Durante la realización de la presente tesis se ha obtenido las curvas de carga del sector residencial del cantón Cuenca basadas en la información del Departamento de Calidad de la Dirección de Planificación, en estas mediciones se encuentra gran cantidad de información que sirvió para determinar las curvas de carga de los diversos sectores con un estimable margen de confianza.

Esta información ha sido contrastada con la investigación "Factores de Responsabilidad 
de Carga”, elaborada en 1999, evidenciándose la variación de ciertos comportamientos de consumo, entre ellos:

- Aumento de los equipos de computación.

- Aumento en el uso de lámparas fluorescentes en los estratos de menor consumo.

- Reducción en el porcentaje de uso de calentadores de agua eléctricos, ampliándose el margen de uso de calentadores que queman GLP.

Al igual que en la caracterización de carga de 1999 se fusionaron los estratos 1 hasta el estrato 4, ya que su consumo es muy bajo.

La campaña de uso de LFC ha sido muy favorable para los estratos de menor consumo, pero estas lámparas no son usadas mayoritariamente en los estratos de mayor consumo. Una de las principales barreras detectadas se basa en la opinión del cliente que considera estas lámparas como 'antiestéticas' o de colores 'pálidos'. Un problema importante se presenta por el uso de sistemas atenuables en estos estratos, lo cual hace incompatibles las LFC debido a la imposibilidad de atenuación de las lámparas que se puede conseguir en el mercado.

El consumo en stand by o modo de espera representa cerca del 4\% del consumo en el sector residencial del cantón Cuenca. Esta pérdida de energía puede ser reducida mediante la desconexión de los aparatos eléctricos instalando regletas con interruptores.

La T.V. es un equipo de presencia muy significativa en el cantón Cuenca, aproximadamente existen entre 3 y 4 receptores de T.V. por cada familia, este equipo se mantiene en- cendido durante largos periodos, incluso cuando no está siendo vista representando un 8\% del consumo diario.

La categoría de equipos de computación representa un $17 \%$ del consumo diario, las computadoras de escritorio tienen un consumo elevado en este grupo, el aumento en el uso de computadoras portátiles de bajo consumo que sustituyen a las unidades de escritorio favorece una reducción de consumo.

El calentamiento de agua representa un 6\% de la energía consumida por el sector residencial, esta categoría ha sido analizada llegándose a las siguientes posibles soluciones:

\subsubsection{Sustitución de calentadores eléctricos por calentadores termo-solares}

Esta alternativa requiere una inversión elevada, pero es recuperada al quinto año reduciéndose el consumo eléctrico y el uso de GLP, con un beneficio económico y ambiental.

\subsubsection{Sustitución de calentadores eléctricos por calentadores que consumen GLP}

Esta alternativa es más económica que la anterior, evita el consumo de energía eléctrica pero usa como combustible GLP, en las actuales condiciones del país, el GLP tiene un subsidio que abarata su costo, pero representa un fuerte egreso para el Estado.

En caso de sustituir el calentador eléctrico por uno de GLP, la inversión se recupera en el tercer año de funcionamiento, pero se crea una nueva molestia: la sustitución de los tanques de combustible. 


\subsubsection{Sustitución de calentadores que consumen GLP por calentadores termo-solares}

Al reemplazar 2000 calentadores de agua en que queman GLP por calentadores solares se evita producir 212,55 Ton de CO2 mensualmente, si se participa en los mercados de carbono esta reducción puede representar un ingreso económico de 5313,75 USD mensuales por la venta de bonos de carbono.

\subsection{Sustitución de electrodomésticos por equipos más eficientes}

La refrigeración constituye el equipo eléctrico de mayor consumo en el sector residencial, con un $27 \%$ del consumo diario, por lo cual se han planteado alternativas de sustitución de equipos que lleguen al final de su vida útil. Un equipo de mayor eficiencia tiene un costo inicial mayor, pero la diferencia con respecto a un equipo menos eficiente se ve compensada con su reducción en el consumo.

\subsubsection{Cargos por transmisión}

Aplicando la demanda eléctrica en la hora pico es posible reducir 12197,231 kW, que equivale aproximadamente a una reducción del 9,4 \% en la demanda en hora pico, con lo cual la máxima demanda disminuye y consecuentemente el monto de la TFTT es reducido en alrededor de USD \$ 19.027,68 mensuales, este valor fue calculado con los valores a enero de 2009.

\subsubsection{Retraso de inversiones en expansión del Sistema de Subtransmisión y Distribución}

Aplicando el PGD es posible reducir el consumo en la hora pico. Para el cálculo del aho- rro en las inversiones se hicieron las siguientes consideraciones:

- Las ampliaciones de red solo se realizan para satisfacer la máxima demanda (hora pico).

- Se descontó el valor de las anualidades de los activos de líneas y subestaciones de subtransmisión.

- El monto de las inversiones para ampliaciones en el sector residencial fue calculado en base al consumo del sector frente al consumo total de la CENTROSUR.

- El monto de las inversiones para el cantón Cuenca se obtuvo en proporción al número de clientes del cantón con respecto al total de la CENTROSUR.

\subsection{Plan de gestión de la demanda eléctrica}

La implementación de un PGD significa una reducción en la venta de energía, sin embargo los beneficios que obtenga serán: económicos y de imagen corporativa, ya que se presentará a la CENTROSUR comprometida la conservación del planeta al plantear a sus clientes una estrategia para combatir el cambio climático.

Las alternativas presentadas para reducir el consumo en el sector residencial del cantón Cuenca planteadas en esta investigación son:

- Campaña informativa.

- Sustitución de equipos eléctricos.

- Sustitución de calentadores solares.

- Participación de la CENTROSUR en el mercado de bonos de carbono. 
Con la implementación de las medidas mencionadas en la presente investigación los clientes residenciales de la CENTROSUR pueden reducir el consumo de energía eléctrica entre un 5 y 30\% lo que significa un ahorro económico para el cliente modificando sus hábitos de consumo y con una inversión moderada.

\section{Recomendaciones}

Es importante que la CENTROSUR implemente una oficina de eficiencia energética que administre y efectúe programas de uso eficiente de energía y de continuidad a la investigación en esta área.

En la presente investigación se plantea introducción de sistemas de calentamiento solares, participación en el mercado de bonos de carbono, estos temas deben ser abordados en futuras investigaciones.

La CENTROSUR debe iniciar un acercamiento hacia el consumidor, presentándole alternativas para reducir su consumo de energía: además, como una acción complementaria, se puede crear una dependencia que realice 'auditorias energéticas', que permitan certificar el nivel de eficiencia energética de grandes consumidores.

A nivel educativo se realizan las siguientes recomendaciones:

- Realizar invitaciones o concursos de tesis de pre y posgrado, en temas de eficiencia energética.

- Incorporar en los niveles iníciales de educación campañas de 'cultura de ahorro de energía' esto permitirá preparar a los más jóvenes para que consuman energía de manera más adecuada.
- En la educación superior se podrían establecer convenios para la capacitación de profesionales en áreas de eficiencia energética, esto además de ser beneficioso para los profesionales y la colectividad permitirá un aporte a la situación medioambiental.

Para lograr que los usuarios residenciales realicen su consumo de manera eficiente la CENTROSUR debe:

- Definir acciones para ingresar al mercado de bonos de carbono, esta es una nueva oportunidad de negocio, que podría financiar estas propuestas.

- Realizar la importación y distribución de LFC atenuables para incentivar el uso de estos equipos en los estratos de mayor consumo de energía.

- Incentivar el uso de regletas con interruptores para desconectar los equipos eléctricos mientras no se usan, para ello la CENTROSUR puede realizar la distribución gratuita de regletas para los clientes.

Una de las alternativas para disminuir el consumo en las horas de mayor demanda es reducir el uso de un equipo de T.V. en cada hogar. Otra alternativa es el uso centralizado de la T.V., que permitirá reunir a la familia en un solo ambiente, presentando un ahorro de energía y permitiendo un momento de socialización que mejore las relaciones familiares.

Es necesario realizar un análisis de la influencia de los equipos electrónicos y lámparas fluorescentes instalados en el sistema CENTROSUR y su influencia en la calidad de energía. ||||||| 


\section{Referencias bibliográficas}

\section{Textos}

[1] CD con las mediciones realizadas por el Departamento de Calidad de la Dirección de Planificación de la E.E.R.C.S. C.A, discos de enero de 2006 hasta diciembre de 2008.

[2] E.E.R.C.S. C.A., Factores de responsabilidad de la carga en la EERCS CA, Cuenca, 1999.

[3] Hunt, Sally, Making Competition work in Electricity; Wiley \& Sons; New York, 2002.

[4] Willis, H Lee, Spatial Electric Load Forecasting, Nueva York: Editorial Marcel Dekker, 1996.

[5] Horning, M., Guía de mantenimiento del transformador, Nueva York: Transformer Maintenance Institute (TMI), 2005.

[6] Lind, D; Marchal, W; Mason, R†; Estadística para Administración y Economía, Madrid: McGraw Hill, 2006.

[7] Díaz, Patricio y Carlos Romero, Caracterización de la carga para un programa de Administración de Demanda en el Sector Residencial Urbano de la Ciudad de Cuenca, Tesis previa a la obtención del título de Ingeniero Eléctrico, Cuenca: Universidad de Cuenca-EERCS CA, 1998.

[8] Morales, Diego, Cálculo de la Energía no suministrada usando al GIS como herramienta Informática en la Empresa Eléctrica regional Centro Sur C.A, Tesis previa a la obtención del título de Ingeniero Eléctrico, Cuenca: Universidad Politécnica Salesiana-EERCS CA, 2009.

[9] Vanegas, Jaime, Energía Térmica-Solar para el calentamiento de agua en la ciudad de Cuenca, Tesis de maestría, Cuenca: Universidad de Cuenca, Cuenca, 2007.

\section{Documentos técnicos}

[1] Bonneville, Eric, PhD; Rialhe, Anne, "Demand Side Management for residential and commercial end-users"; AERE, Francis, 2007.

[2] CENACE, "Estadística del sector eléctrico Ecuatoriano primer semestre del año 2008”, Quito, 2008.

[3] CONELEC, "Plan Maestro de Electrificación del Ecuador 2007-2016”, Quito, 2006.

[4] Eguíluz, L. I.; J. C. Lavandero; M. Mañana, M.; P. Sánchez "Eficiencia Energética Y Calidad de Suministro Eléctrico”; Dpto. de Ingeniería Eléctrica y Energética, Universidad de Cantabria, 2006.

[5] Environment Protection Agency, "Report 2005", Washington, 2006.

[6] International Energy Agency (IEA), "INDEEP Analysis Report”; París, 2004.

[7] M. Torrent y M.A. Larrayoz; Gestión de la demanda eléctrica, un método eco energético, Dpto. Ingeniería Eléctrica. Universidad Politécnica de Cataluña, 2006.

[8] Ministerio de Electricidad y Energías Renovables, Políticas y estrategias para el cambio de la matriz energética del Ecuador, Quito, 2008.

[9] Ministerio de Energía y Minas, Eficiencia Energética, Proyecto Guardián de Luz; Quito, 2006.

[10] Oficina Ecuatoriana de Promoción del MDLCORDELIM; "Mecanismos de Desarrollo Limpio en Ecuador: Un diagnóstico rápido de los retos y oportunidades en el Mercado de Carbono”; Quito, 2006.

[11] Proyecto Alpheo, Guide Alpheo pour la maitrise de l'energie et de l'eau, Francia, 2005. 